\title{
ORIGINALES
}

\section{Gestación adolescente y su repercusión en el recién nacido. Estudio de casos y controles}

\author{
E. Dorta ${ }^{a}$, J. Molina ${ }^{a}$, J.A. García-Fernández ${ }^{\mathrm{b}}$ y L. Serra ${ }^{\mathrm{c}}$ \\ a Unidad de Apoyo a la Investigación. Complejo Hospitalario Materno-Insular. Las Palmas de Gran Canaria. \\ 'Servicio de Gineco-Obstetricia. Hospital Universitario Materno Infantil de Canarias. Las Palmas de Gran Canaria. \\ 'Medicina Preventiva. Universidad de Las Palmas de Gran Canaria. Las Palmas de Gran Canaria. España.
}

\section{ABSTRACT}

Objective: To know the principal characteristics associated to adolescent pregnancy and value it as a possible risk factor for low birthweight.

Materials and methods: To study cases and controls for six months. The cases were selected from nuliparous women aged from 14 years to 19 years admitted to obstetrics during labour. The controls, nuliparous women aged 20 years or more, were admitted to the same center during the same period of time and were chosen randomly from the admissions book. These were coded and later processed using the data of the SPSS V 11.0 programme; descriptive analysis of the data was made, OR was calculated as the measurement of association, with an CI of 95\% and the Chi squared test as a contrast of the hypothesis, and assuming the value $\mathrm{p}<0.05$ to be the statistically significant difference between them. A multivariant analysis was also carried out using a multiple logistic regression model.

Results: 242 cases and 484 controls. The risk factors associated with low birth weight identified in the analysis were: the age of the mother $<19$ years OR 2.12; CI (1.30-3.44) and antenatal control $<5$ visits, OR 5.15 (2.81-9.41). Generally speaking the adolescent mothers were out of the education system and mostly worked as domestic assistants, they also had a higher incidence of low birth weight and prematurity.

Conclusions: There is an association between the age of the mother and low weight neonates, which increases amongst those who do not attend the antenatal clinics regularly.

Aceptado para su publicación el 12 de enero de 2004.

\section{INTRODUCCIÓN}

La Organización Mundial de la Salud define la adolescencia como el período de la vida en que el individuo adquiere la madurez reproductiva y transita por los patrones psicológicos de la niñez a la vida adulta, y fija sus límites entre los 10 y los 19 años ${ }^{1}$. Conforme a las distintas edades, la adolescencia se divide en 3 etapas: temprana (10-13 años), media (14-16 años) y tardía (17-19 años). Para entender las actitudes de las jóvenes, especialmente durante un embarazo, es importante conocer las características de estas etapas, sabiendo que una adolescente embarazada se comportará como corresponde al momento de la vida que está transitando, y no madurará a etapas posteriores por el simple hecho de estar embarazada $^{2}$.

Distintos autores afirman que la gestación adolescente es una condición de alto riesgo ${ }^{3-4}$. Otros señalan que estas embarazadas siguen con frecuencia una dieta inadecuada y acceden a la atención prenatal en un estadio muy avanzado de la gestación ${ }^{4-5}$, ya que existe una tendencia de la joven a ignorar su situación, bien sea por miedo a la desaprobación familiar o porque a esta edad se es más proclive a demostrar comportamientos arriesgados y se tiene poca conciencia sobre la salud. Los recién nacidos de estas jóvenes son el producto de un embarazo con una mayor probabilidad de tener complicaciones, entre las que destacan el retraso del crecimiento intrauterino, la prematuridad, el bajo peso al nacer ${ }^{5-7}$, el retraso en el desarrollo, la anemia ferropénica en el primer año de vida e incluso un mayor riesgo de sufrir maltrato físico ${ }^{8,9}$.

El embarazo y la maternidad a edades tempranas no es patrimonio de los países del tercer mundo, es un problema universal que despierta un creciente interés en los países desarrollados, no sólo por la mortalidad que origina sino también por la magnitud de 
TABLA I. Características sociodemográficas de las embarazadas estudiadas en el Hospital Universitario Materno Infantil de Canarias entre enero y agosto de 2002

\begin{tabular}{|c|c|c|c|c|c|}
\hline CARACTERÍSTICAS & ADOLESCENTES $(\mathrm{n}=242)$ & ADULTAS $(n=484)$ & ODDS RATIO & IC DEL 95\% & $\mathrm{p}$ \\
\hline \multicolumn{6}{|l|}{ Escolaridad } \\
\hline Analfabeta & $33(13,6 \%)$ & $8(1,0 \%)$ & \multirow[t]{2}{*}{9,39} & \multirow{2}{*}{$4,07-22,46$} & \multirow{2}{*}{$<0,01$} \\
\hline EGB-COU-UNIV & $209(86,3 \%)$ & $476(98,3 \%)$ & & & \\
\hline \multicolumn{6}{|l|}{ Ocupación } \\
\hline Remunerada & $32(13,2 \%)$ & $336(69,3 \%)$ & \multirow[t]{2}{*}{14,90} & \multirow{2}{*}{$9,62-23,19$} & \multirow{2}{*}{$<0,01$} \\
\hline No remunerada & $210(86,8 \%)$ & $148(30,6 \%)$ & & & \\
\hline \multicolumn{6}{|l|}{ Convivencia } \\
\hline Compañero & $218(30,02 \%)$ & $481(66,3 \%)$ & \multirow[t]{2}{*}{17,65} & \multirow{2}{*}{$5,0-74,37$} & \multirow{2}{*}{$<0,01$} \\
\hline Padre desconocido & $24(3,3 \%)$ & $3(0,4 \%)$ & & & \\
\hline \multicolumn{6}{|l|}{ Ocupación del padre } \\
\hline No remunerada & $36(4,9 \%)$ & $21(2,8 \%)$ & \multirow{2}{*}{4,15} & \multirow{2}{*}{$2,28-7,58$} & \multirow{2}{*}{$<0,01$} \\
\hline Ocupación remunerada & $188(75,8 \%)$ & $455(62,6 \%)$ & & & \\
\hline \multicolumn{6}{|l|}{ Escolaridad del padre } \\
\hline Analfabeto & $39(16,1 \%)$ & $15(3,0 \%)$ & \multirow[t]{2}{*}{13,33} & \multirow[t]{2}{*}{$7,72-23,42$} & \multirow[t]{2}{*}{$<0,01$} \\
\hline EGB-COU-UNIV & $200(82,6 \%)$ & $465(96,1 \%)$ & & & \\
\hline
\end{tabular}

la morbilidad, que tanto a corto como a largo plazo le acompañan, y envuelven a la madre y al niño. Canarias es una población de las más jóvenes de España, lo que refuerza la importancia de estudiar las características del embarazo y el parto en jóvenes entre 14 y 19 años. Por tanto, nos planteamos como objetivos llevar a cabo un estudio comparativo referente a las complicaciones obstétricas y al tipo de parto de las adolescentes contrastadas con gestantes adultas, así como la valoración de la maternidad a esa edad como posible factor de riesgo para el bajo peso al nacer.

\section{MATERIAL Y MÉTODOS}

El diseño propuesto fue observacional de casos y controles, entre enero y agosto de 2002. Se incluyó al total de gestantes en edad fértil que finalizaron el embarazo en la sala de partos del Hospital Universitario Materno Infantil de Canarias. Los casos procedían de la identificación de todas las pacientes nulíparas con edades comprendidas entre los 14 y los 19 años que iban ingresando en el servicio de obstetricia por trabajo de parto y dieron a luz un recién nacido vivo, durante el tiempo asignado para el estudio.

Para la elección de los controles se escogió a embarazadas nulíparas de 20 años o más que ingresaron por trabajo de parto, y al igual que los casos concibieron un recién nacido vivo. Para cada caso se localizaron 2 controles, que se seleccionaron a partir de las primeras 2 mujeres que aparecían en el libro de registros de parto. Los antecedentes obstétricos se tomaron como criterio de exclusión, y las pacientes con más de un embarazo se descartaron del estudio. Los datos se recolectaron a través de la revisión de la historia clínica obstétrica, y se procedió a la codificación y posterior procesamiento de los datos por medio del programa SPSS v. 11.0. El tratamiento estadístico constó de los siguientes apartados:

1. Análisis descriptivo de los datos, mediante el cálculo de frecuencias absolutas y relativas en porcentaje.

2. Estadística inferencial, con cálculo de la odds ratio (OR), como medida de asociación entre las variables con un intervalo de confianza (IC) del 95\%. Se utilizó el test de la $\chi^{2}$ como test de hipótesis, asumiendo como diferencias estadísticamente significativas aquellas en que el valor de $\mathrm{p}$ fuera inferior a 0,05 .

3. Análisis multivariante mediante un modelo de regresión logística múltiple, teniendo como variable de respuesta el bajo peso al nacer.

\section{RESULTADOS}

Entre enero y agosto de 2002 se atendieron 3.757 partos, de los cuales $258(6,86 \%)$ correspondieron a madres adolescentes. Tras aplicar los criterios de exclusión, el número total de casos fue de 242. La edad media de este grupo de primigestas adolescentes fue de 17,6 años, y la de los controles, de 28,09. La distribución de estos casos por edad se contabilizó de la siguiente forma: 2 de 14 años, 10 de 15, 36 de 16, 47 de 17,70 de 18 y 77 de 19 .

En la tabla I se muestran las características de las variables estudiadas; cabe resaltar que un 13,6\% de los casos eran analfabetas, y un 86,3\% tenía estudios de EGB o COU. En los controles se clasificó como analfabeta el 1,0\% de la muestra, y el 44,2\% había 
Dorta E, et al. Gestación adolescente y su repercusión en el recién nacido. Estudio de casos y controles

TABLA II. Diferencias entre las características obstétricas y perinatales de gestantes adolescentes y adultas

\begin{tabular}{|c|c|c|c|c|c|}
\hline VARIABLES & ADOLESCENTE & ADULTA & ODDS RATIO & IC DEL 95\% & $\mathrm{p}$ \\
\hline \multicolumn{6}{|l|}{ Control prenatal } \\
\hline$<5$ consultas & $95(17,4 \%)$ & $82(3,4 \%)$ & 6,02 & \multirow{2}{*}{$3,31-10,97$} & \multirow{2}{*}{$<0,01$} \\
\hline$\geq 5$ consultas & $147(82,6 \%)$ & $393(96,6 \%)$ & 1 & & \\
\hline \multicolumn{6}{|l|}{ Edad gestacional } \\
\hline Pretérmino & $32(13,2 \%)$ & $30(6,2 \%)$ & 2,31 & \multirow[t]{2}{*}{$1,37-3,90$} & \multirow[t]{2}{*}{$<0,01$} \\
\hline A término & $210(86,2 \%)$ & $454(93,8 \%)$ & 1 & & \\
\hline \multicolumn{6}{|c|}{ Peso del recién nacido } \\
\hline Bajo peso & $53(21,9 \%)$ & $41(8,5 \%)$ & 3,03 & \multirow[t]{2}{*}{$1,95-4,71$} & \multirow[t]{2}{*}{$<0,01$} \\
\hline Peso normal & $189(78,1 \%)$ & $443(91,5 \%)$ & 1 & & \\
\hline \multicolumn{6}{|l|}{ Apgar } \\
\hline$<6$ & $19(7,9 \%)$ & $34(7,0 \%)$ & 1,13 & \multirow[t]{2}{*}{$0,63-2,02$} & \multirow[t]{2}{*}{0,69} \\
\hline$\geq 6$ & $223(92,1 \%)$ & $449(93,0 \%)$ & 1 & & \\
\hline
\end{tabular}

IC: intervalo de confianza.

cursado estudios de EGB. Al comparar la ocupación de la madre, encontramos diferencias estadísticamente significativas entre ambos grupos $(\mathrm{OR}=14,90) . \mathrm{El}$ $30,6 \%$ del total de las adultas estudiadas no tenía una ocupación remunerada frente al $86,8 \%$ en las menores de 19 años.

El 55,8\% de las gestantes adolescentes se dedicaba a las labores del hogar, situación que se percibió en el $20,7 \%$ del grupo de 20 años o más. Un 13,2\% de los casos y un $8,5 \%$ de los controles declararon que se encontraban en el paro; sólo un $17,81 \%$ de las adolescentes aparecían registradas como estudiantes. Un $13,2 \%$ de los casos trabajaba en una ocupación remunerada, lo que se advierte en el 30,6\% del grupo control.

En la tabla II se expone que las gestantes adolescentes frecuentan menos las consultas prenatales, en comparación con las adultas ( $\mathrm{p}<0,05$ para el rango etario entre 14 y 19 años), y se advierte que los embarazos son mal controlados en el $17,4 \%$ de los casos y en el $3,4 \%$ de los controles. A su vez se observa que existe una asociación estadísticamente significativa entre el embarazo a edades tempranas y el riesgo de presentar bajo peso al nacer, y la prematuridad.

En la tabla III se muestran los resultados del análisis de regresión logística que explican el bajo peso al nacer en el Hospital Universitario Materno Infantil de Canarias, y destacan como principales factores de riesgo la edad materna menor de 20 años y la falta de un control prenatal adecuado, que se advierte como la variable que mejor expresa esta variable $(\mathrm{OR}=5,15)$.

\section{DISCUSIÓN}

Se eligió un estudio de casos y controles, ya que es un diseño que permite la evaluación de múltiples factores, es ideal para acontecimientos poco comunes o
TABLA III. Factores de riesgo para bajo peso al nacer: análisis de regresión logística

\begin{tabular}{l|c|c|c}
\hline & $\mathrm{P}$ & ODDS RATIO & IC DEL 95\% \\
\hline Edad de la madre & 0,002 & 2,12 & $1,30-3,44$ \\
Control prenatal & 0,000 & 5,15 & $2,81-9,41$ \\
Recién nacido pretérmino & 0,000 & 5,37 & $2,92-9,89$ \\
Constante & 0,135 & 0,54 & \\
\hline
\end{tabular}

IC: intervalo de confianza. Variable/s excluida/s del modelo final: escolaridad de la madre y tipo de parto.

con períodos de inducción largos. Con este diseño los errores sistemáticos son más probables, especialmente los de selección y de información, lo que hay que tener en cuenta a la hora de valorar los hallazgos de nuestra investigación, ya que los datos fueron tomados de las historias clínicas, fuente de información que puede estar sujeta a estos sesgos ${ }^{10}$.

Se ha comparado a 242 madres entre 14 y 19 años con 484 controles adultas, y el perfil de adolescentes encontrado es similar al de otros estudios ${ }^{11,12}$ (solteras, dedicadas a las labores domésticas y fuera del sistema educativo). Valero et $\mathrm{al}^{13}$ observaron esta característica en un $67,3 \%$ de sus pacientes. Cabe destacar que sólo un $17,81 \%$ de las jóvenes aún estudia, lo que representa una proporción muy baja, similar a la hallada por Oliveira ${ }^{14}$, que advirtió que un $21,1 \%$ de las jóvenes aún continuaba su formación académica. Mott y Marsiglio ${ }^{15}$ describieron que una elevada proporción de madres adolescentes abandonaba los estudios por motivo del embarazo, lo que hacía muy difícil su reincorporación después del parto.

El 13,2\% de los casos fueron partos pretérmino, cifra que coincide con el $12 \%$ descrito por Cornelius et $\mathrm{al}^{7}$. Monterrosa y Bello ${ }^{3}$ encontraron que el $7,3 \%$ de 10.550 partos fueron prematuros, y de éstos, $36,4 \%$ 
ocurrieron en jóvenes entre los 14 y los 18 años. Aunque varios autores proponen que se estandarice la edad materna como un factor que predispone al parto pretérmino en edades menores a los 18 años $^{4,16,17}$, en la literatura a médica se encuentran variaciones en cuanto a la existencia de un riesgo intrínseco relativo a que la edad cronológica podría alterar los resultados perinatales.

Newman ${ }^{18}$, y Cunninghan y Lindheirmer ${ }^{19}$ coinciden en considerar que el estrés es un factor desencadenante del trabajo de parto antes de la semana 37, y argumentan que una adolescente embarazada podría ser más propensa a sus efectos debido a la incertidumbre o a los temores sobre su integridad física en el momento del parto. Otros investigadores ${ }^{18-20}$ han señalado también la hipótesis de la inmadurez biológica y una adaptación uterina ineficiente.

Muchos autores afirman ${ }^{4,21-23}$ que la tasa de recién nacidos de bajo peso en embarazadas adolescentes es mayor que la hallada en mujeres adultas. Sin embargo, hemos revisado algunas publicaciones que consideran contradictoria la influencia de la edad materna en el peso del neonato, y especulan sobre la presencia de variables sociodemográficas asociadas a la edad de la gestante ${ }^{24}$. Nuestros resultados han demostrado que un $21,9 \%$ de los recién nacidos tenía un peso menor al límite inferior de la normalidad, porcentaje similar al obtenido por Kennely et $\mathrm{al}^{25}$, que hallaron cifras entre un 15 y un $20 \%$.

Strobino et $\mathrm{al}^{26}$ estudiaron 3 diferentes hipótesis para explicar el bajo peso al nacimiento registrado en los hijos de adolescentes en Estados Unidos (desventaja social, inmadurez biológica y comportamiento no saludable durante la gestación), y encontraron que el ambiente de privación social era el factor más relevante a la hora de explicar sus causas.

La hipertensión arterial inducida por el embarazo es una complicación frecuente en menores de 17 años $^{18}$. Si bien los mecanismos implicados sobre el peso fetal no están bien aclarados, es posible que el marcado aumento de la resistencia vascular periférica que presentan las madres hipertensas interfiera en la circulación placentaria y, por ende, en el normal desarrollo del feto. Monterrosa y Bello ${ }^{3}$ hacen referencia a que, en la revisión de 9.000 casos de pacientes con eclampsia, el $37,2 \%$ tenía entre 14 y 18 años. Distintos autores ${ }^{3,22}$ señalan que la hipertensión arterial inducida por el embarazo es cinco veces más frecuente en menores de 17 años, y lo atribuyen al insuficiente desarrollo endocrino y biológico para establecer la adaptación a la gestación.

Se ha sugerido la importancia del control prenatal como un factor de prevención de complicaciones para la madre y su futuro hijo ${ }^{27}$. En nuestro estudio esta fue una variable importante, como puso de manifiesto el análisis multivariante (tabla III), lo que coincide con Kogan et a ${ }^{28,29}$, que concluyeron que los cuidados prenatales disminuían la incidencia de recién nacidos pretérmino.

Pardo y Uriza ${ }^{16}$, en un estudio que agrupó 11 instituciones y en el que se evaluó a 3.364 adolescentes de 54.497 partos, hallaron que el $27,5 \%$ de las jóvenes tenía un control prenatal inferior a las 6 consultas asistidas. Fraser et $\mathrm{al}^{4}$ aseveran que existe una relación inversamente proporcional entre el control prenatal y la morbimortalidad materna y/o perinatal. También debemos tener en cuenta la mayor prevalencia de hábitos tóxicos y transgresiones dietéticas ${ }^{7,30}$.

En nuestro trabajo el porcentaje de partos distócicos en los casos fue del 20,6 y en los controles de un 19,2\%. En Estados Unidos el porcentaje de partos por cesáreas en menores de 19 años fue de un 7\% para $2001^{31}$. Si bien no encontramos una asociación significativa entre el control prenatal y la forma de terminación del parto, Smith y Pell21, en Escocia, concluyeron que en las adolescentes sin un adecuado seguimiento del embarazo había una mayor frecuencia de cesáreas y una mayor necesidad de fórceps. Montgomery ${ }^{32}$ expone que lo que más influye en la forma en que se presenta el parto es la actitud de la madre adolescente, y que el deseo de embarazo en las jóvenes tiene una relación directamente proporcional con la forma de terminación de éste. Este autor ha encontrado que un $15 \%$ de los embarazos a esta edad son deseados y planeados, y asocia estos resultados a una repetición de patrones familiares y a una situación social más deprimida.

El índice de Apgar en el primer minuto determina la necesidad inmediata de asistencia ventilatoria. En publicaciones revisadas en que se ha incluido esta variable se afirma que las puntuaciones en las valoraciones de los recién nacidos de madres adolescentes son inferiores a las encontradas en hijos de madres adul$\operatorname{tas}^{7,17,33}$. En nuestra muestra la frecuencia de una baja puntuación en esta prueba fue similar en casos y controles, y no encontramos diferencias significativas entre ambos $(\mathrm{OR}=1,13$; IC del 95\%, 0,63-2,02). Este resultado concuerda con un trabajo efectuado por Escobar y Balcazar ${ }^{33}$ en el que la estimación de la OR para un Apgar menor de 6 en las adolescentes no proporcionó información sobre la existencia de una asociación. Amini y Catalano ${ }^{27}$ exponen que la depresión al nacer, tanto al minuto como a los $5 \mathrm{~min}$, tiene como factores de riesgo relevantes ser madre soltera o analfabeta.

Nuestro estudio pone de manifiesto que, de una forma general, las madres jóvenes están fuera del sis- 
Dorta E, et al. Gestación adolescente y su repercusión en el recién nacido. Estudio de casos y controles

tema educativo y, en su mayoría, dedicadas a las labores del hogar, hay una mayor prevalencia de nacimientos de bajo peso y prematuridad entre las adolescentes $\mathrm{y}$, generalmente, tienden a asistir de una forma irregular a las consultas prenatales. Este hecho es motivo de preocupación por la tendencia al aumento de gestantes en este grupo etario, lo que podría incrementar el número de recién nacidos con mayor morbimortalidad.

El análisis multivariante identificó que hay una asociación entre la edad de la madre y el bajo peso del recién nacido, que se incrementa entre aquellas que asisten a un número limitado de consultas prenatales. Esto nos ha hecho concluir sobre el efecto protector del control prenatal en relación con el bajo peso al nacer. En busca de explicaciones detalladas sobre los mecanismos que permiten esta asociación, creemos que las condiciones y los estilos de vida, como la alimentación de la adolescente durante la gestación, pasando por el soporte familiar y social, hasta las particularidades de la comunidad, deben ser objeto de futuras investigaciones para delinear estrategias objetivas que reduzcan las desigualdades en el bajo peso, la morbilidad perinatal y otros resultados adversos, no sólo desde el punto de vista biológico, sino también desde el psicológico y social, que disminuyen la calidad de vida del recién nacido.

\section{RESUMEN}

Objetivo: Conocer las principales características del embarazo en la adolescencia y valorarlo como posible factor de riesgo para el bajo peso al nacer.

Materiales y métodos: Estudio de casos y controles de 6 meses de duración. Los casos se seleccionaron a partir de nulíparas, de edades comprendidas entre los 14 y los 19 años, que iban ingresando en el servicio de obstetricia por trabajo de parto. Los controles, nulíparas de 20 años o más, que ingresaron en el mismo centro y en el mismo período, se escogieron de forma aleatoria del libro de admisiones. Se procedió a la codificación y al posterior procesamiento de los datos con el programa SPSS v. 11.0. Se llevaron a cabo un análisis descriptivo de los datos, y un cálculo de la odds ratio como medida de asociación, con un intervalo de confianza del $95 \%$ y un test de la $\chi^{2}$ como contraste de hipótesis, asumiendo como diferencias estadísticamente significativas las que tenían un valor de $\mathrm{p}$ $<0,05$. Asimismo, se realizó análisis multivariante mediante un modelo de regresión logística múltiple.

Resultados: Doscientos cuarenta y dos casos y 484 controles. Los factores de riesgo asociados al bajo peso al nacer identificados en el análisis fueron: edad de la madre $<19$ años (odds ratio $=2,12$; intervalo de confianza del $95 \%, 1,30-3,44)$ y control prenatal $<5$ consultas (odds ratio $=5,15$ intervalo de confianza del $95 \%, 2,81-9,41)$. De forma general, las madres adolescentes están fuera del sistema educativo, en su mayoría dedicadas a las labores del hogar, y en ellas existe una mayor prevalencia de bajo peso y de prematuridad.

Conclusiones: Existe asociación entre la edad de la madre y el bajo peso del neonato, que se incrementa entre quienes asisten de forma irregular al control prenatal.

\section{BIBLIOGRAFÍA}

1. OMS/UNICEF. Declaración conjunta. Higiene de la reproducción en la adolescencia. Ginebra: Organización Mundial de la Salud, 1989.

2. Issler J. Embarazo en la adolescencia. Rev de Postgrado Cátedra 6 de Med 2001;107:11-23.

3. Monterrosa A, Bello A. Atención obstétrica en adolescentes. Rev Col Obstet Ginecol 1996;47:15-22.

4. Fraser AM, Brokert JE, Ward RH. Association of young maternal age with adverse reproductive outcomes. N Engl J Med 1995;332:1113-7.

5. Gripp G, Azevedo A. A gravidez na adolescencia e fator de risco para o baixo peso ao nacer. Rev Chil Pediatr 2000;71: 516-22.

6. Villanueva LA, Pérez,MM, Martínez H, García E. Características obstétricas del embarazo en la adolescencia. Ginecol Obstet Mex 1997;67:356-60.

7. Cornelius M, Goldsmidt, Taylor PM. Prenatal alcohol abuse among teenagers: effects on neonatal outcomes. Alcohol Clin Exp Res 1999;23:1238-44.

8. American Academy of Pediatric, Committee on Adolescence. Adolescents pegnancy. Currents trends and issues. Pediatrics 1999;103:516-20.

9. Cooper L, Leland N, Alexander G. Effet of the maternal age on birth outcomes, among young adolescents. Clin Obstet Ginecol 1996;87:83-8.

10. Escuela Andaluza de Salud Pública. Metodología de investigación y escritura científica en clínica. $3{ }^{a}$ ed. Granada: Escuela Andaluza de Salud Pública, 1998.

11. Doval J, Blanco S, Vizcaíno M, Álvarez E, Rodríguez R, González M. Embarazo en adolescentes. Variables clínicas y sociales. Acta Ginecol 1995;52:9-14.

12. Portella E, Catillo A. Aspectos sociales del embarazo en adolescentes. Gac Sanit 1988;6:150-3.

13. Valero C, Nebot M, Villalbi J. Embarazo en adolescentes: Distribución, antecedentes y consecuencias. Gac Sanit 1994; 43:155-61.

14. Oliveira M. Maes adolescentes. $1 .^{\mathrm{a}}$ ed. Lisboa: Barca Nova, 1993.

15. Mott F, Marsiglio W. Early chidbearing and completion of high school. Fam Plan Persp 1985;17:234-7.

16. Pardo F, Uriza G. Estudio de embarazo adolescente en 11 instituciones. Rev Col Obst Ginecol 1997;42:109-21.

17. Bashado S, Daren O. Prenatal prediction of neonatal outcome in the extremly low-birth weight infant. Am J Obstet Gynecol 1998;178:462-8. 
18. Newman RB. Risk factors for neonatal in the state of South Carolina. Am J Obstet Gynecol 1999;180:757-62.

19. Cunninghan FG, Lindheirmer MD. Hypertension in pregnancy. N. Engl J Med 1992;326:927-32.

20. Olausson P, Crattingius S, Goldenberg R. Determinants of poor pregnancy outcomes among teenagers in Sweden. Obstet Gynecol 1997;89:451-7.

21. Smith G, Pell J. Teenage pregnancy and risk of adverse perinatal outcomes associated with first and second births: population based retrospective cohort study. BMJ 2001;323:476.

22. Lee K, Fergunson R, Corpus M, Gartner L. Maternal age and incidence of low birth weight at term: a population study. Am J Obstet Gynecol 1988;158:84-9.

23. Galan A, Mateos C. Variables perinatales y desigualdades en salud en un área de salud de Cáceres. Gac Sanit 2000;14: 31-8.

24. Creastsas G, Elsheik A. Adolescents pregnancy and its consecuences. Eur J Contracept Reprod Health Care 2002;7: 167-72.

25. Kennely S, Conroy R, Byrne P. Teenage pregnancy in the Rotunda Hospital. Ir Med J 1998;91:209-12.
26. Strobino D, Ensminger M, Kin Y. Mechanisms for maternal age differences in birth weight. Am J Epidemiol 1995;142: 504-14.

27. Amini S, Catalano P. Effect of prenatal care on obstetrical outcome. J Matern Fetal Med 1996;5:142-50.

28. Coll A. Embarazo en la adolescencia. Clin Perinat Arg 1996;4:23-55.

29. Kogan M, Colg R. Comparing mothers report on the content of prenatal care received with recommended national guidelines for care. N Engl J Med 1995;332:1113-7.

30. Loeches M, Catalá E, Orti RM, Mínguez J. Principales factores de riesgo asociados a las gestantes adolescentes de la comunidad Valenciana. Clin Invest Gin Obst 2002;29:2-6.

31. MacDorman MF, Minino AM, Strobino DM, Guyer B. Annual summary of vital statistics-2001. Pediatrics 2002; 110:1037-52

32. Montgomery K. Planned adolescents pregnancy: what they wanted. J Pediatr Health Care 2002;16:282-9.

33. Escobar A, Balcazar N. Factores de riesgo y características de la gestación en la adolescente y su hijo. Rev Col Obst 1995;46:109-16. 